\title{
El patrimonio arquitectónico defensivo giennense: investigación, difusión y puesta en valor. Actuaciones realizadas en las fortalezas que integran la Ruta de los Castillos y las Batallas
}

\author{
Ana María Carmona Casado ${ }^{a}$ \\ á́rea de Historia Medieval, Departamento de Patrimonio Histórico, Universidad de Jaén. Paraje de las Lagunillas, s/n, C.P.23071, \\ Jaén, anamary_cc@hotmail.com
}

\begin{abstract}
Resumen
El presente trabajo ofrece una visión sintetizada sobre el estado actual de una parte de las fortificaciones existentes en la provincia de Jaén, la zona de mayor concentración de estos bienes en toda Europa. Concretamente nos centramos en las integrantes de la Ruta de los Castillos y las Batallas, creada y fomentada desde la Diputación Provincial de Jaén en 2004, y que integra un total de veintiún castillos de diferentes municipios.
\end{abstract}

No es un análisis histórico o morfoestructural de las fortificaciones, sino que parte de tres vértices fundamentales para la conservación de nuestro patrimonio: investigación, restauración y difusión, evaluando su presencia o ausencia en la arquitectura defensiva. Con este análisis seremos conscientes de las debilidades y amenazas que pueden afectar a este patrimonio y que deberemos revitalizar, así como de sus propias fortalezas y las oportunidades, repercutiendo su buen estado en la sociedad como factor de desarrollo.

En la evolución de estos procesos, se han convertido en esenciales la formación y coordinación en los trabajos de equipos multidisciplinares, que sigan el proyecto en todas y cada una de sus fases, donde el diálogo y la coordinación sean el eje central.

Palabras clave: arquitectura defensiva, patrimonio, Alto Guadalquivir, Jaén, investigación, conservación, restauración, difusión.

\begin{abstract}
This article offers a synthesized vision of the current state of a part of the existing fortifications in the province of Jaén, the area of greatest concentration of these in all of Europe. Specifically we focus on the members of the Route of the Castles and Battles, created and promoted from the Provincial Council of Jaén in 2004, and which integrates a total of twenty-one castles from different municipalities.

It is not a historical or morphostructural analysis of the fortifications, but part of three fundamental vertices for the conservation of our heritage: research, restoration and diffusion, evaluating their presence or absence in the defensive architecture. With this analysis we will be aware of the weaknesses and threats that may affect this heritage and that we must revitalize, as well as its own strengths and opportunities, impacting its good status in society as a development factor.

In the evolution of these processes, training and coordination in the work of multidisciplinary teams, which follow the project in each and every one of its phases, where dialogue and coordination are the central axis, have become essential.
\end{abstract}

Keywords: defensive architecture, heritage, Upper Guadalquivir, Jaén, research, conservation, restoration, diffusion. 


\section{Introducción}

Con esta comunicación pretendemos acercar al estudio exhaustivo de las fortificaciones que componen la actual Ruta de los Castillos y las Batallas (Carmona, 2019), realizada por la Diputación de Jaén desde 2004. No se trata de hacer una revisión histórica y morfoestructural de arquitectura defensiva sino profundizar en la dialéctica entre la investigación histórico-arqueológica, la restauración arquitectónica y la difusión final del patrimonio militar (Castillo et al., 1999), determinando en qué fase de estudio se encuentra cada una de ellas. A través de este análisis y valoración, podremos establecer unas pautas de actuación mínimas y comunes a seguir, tanto en las actuales, como en las futuras intervenciones sobre estos bienes, apuntando diversas mejoras que consideremos oportunas.

\section{Contextualización del trabajo}

Jaén es la provincia con mayor concentración de fortificaciones de Europa, condicionado en parte por su localización geográfica al noreste de Andalucía y al sur de una de las entradas naturales: Despeñaperros; y por su evolución histórica que durante siglos conformaron como zona fronteriza este territorio. Concentra 150 fortificaciones medievales (Eslava, 1999), más otras construidas modificando los restos existentes o ex-novo durante la Edad Moderna a modo de castillospalacios, un total de 237 entre castillos, atalayas, torres, murallas... (Madueño, 2017).

\subsection{Contexto histórico}

En la Edad Media, se produjo una completa militarización del espacio, con el avance de los ejércitos cristianos del norte, agudizado este proceso en el contexto de la Batalla de las Navas de Tolosa en 1212 y en la posterior conquista del valle del Guadalquivir hasta la conquista de Granada en 1492. Ese año las fortificaciones del Alto Guadalquivir perdieron definitivamente su función primitiva, siendo abandonadas, utilizadas como canteras en otros edificios, o reutilizándose para nuevos usos como el castillo de Lopera. Los Reyes Católicos prohibieron la construcción de nuevos castillos para evitar la lucha entre las familias nobles, fomentando los cambios de funcionalidad hacia palacios (Alcaudete) u otras formas de hábitat. Las fortificaciones recobraron su función defensiva durante la Guerra de la Independencia (1808-1814) con la ocupación de castillos como el de Santa Catalina o la fortaleza de la Mota, y en el transcurso de la Guerra Civil española (1936-1939) con la reutilización del castillo de Lopera que se encontraba en las cercanías del frente.

\subsection{Antecedentes y estado actual del tema}

Para el estudio de las fortificaciones giennenses son tres los autores que han marcado un antes y un después: Jimena Jurado que dibujó fielmente el estado de algunos de los recintos amurallados provinciales en "Antigüedades del Reino de Jaén" (1639); Morales Talero realizó la primera recopilación en "Castillos y Murallas del Santo Reino de Jaén" (1958); y Eslava Galán con su tesis doctoral sobre "Poliercética y fortificación bajomedieval en el Reino de Jaén" y diferentes publicaciones sobre los Castillos de Jaén. Este legado es recogido, ampliado y enriquecido desde el Área de Historia Medieval de la Universidad de Jaén, bajo la inestimable guía de los catedráticos Vicente Salvatierra y Juan Carlos Castillo, con una apuesta firme por la recuperación de este patrimonio desde enfoques multidisciplinares.

Con el cambio del tipo de Turismo ahora más cultural, y los efectos de la globalización, nació en 2004 la Ruta de los Castillos y las Batallas, desde Castilla La Mancha para adentrarse por Jaén y culminar finalmente en Granada, siendo en nuestra provincia la encargada de su fomento y puesta en marcha la Diputación Provincial de Jaén.

\section{Estudio de las fuentes sobre las fortificaciones}

Como en cualquier construcción tendremos cuatro tipos de fuentes fundamentales: documentales con la investigación en archivos civiles (públicos o privados) y eclesiásticos; bibliográficas con obras monográficas (Pedro de Medina, Argote de Molina, Jiménez Patón, Jimena Jurado, Martínez de Mazas, Madoz...), y publicaciones seriadas (Boletín del Instituto de Estudios Giennenses, Senda de los Huertos: Revista cultural de la provincia de Jaén, Arqueología y Territorio medieval); gráficas a través de dibujos (van der Wyngaerde, Pierre Marie Baldi, Jimena Jurado, Sculp y 
Riudavets...), pinturas (Giménez de la Linde) o fotografías (Romero de Torres, familia Ortega...); y los propios restos de la arquitectura defensiva, con el estudio de la heráldica, de las numerosas marcas de cantería, de los grafitos, o de la epigrafía, siempre con cautela para evitar errores históricos, como ocurrió con la datación del castillo de Baños.

\section{Fortificaciones estudiadas con metodología arqueológica}

Son pocas las fortificaciones investigadas totalmente con metodología arqueológica (Fig. 1), permaneciendo tres castillos a la espera de una mínima actuación: Castro Ferral ( $\mathrm{n}^{\circ}$ 1), en Santa Elena; Navas de Tolosa ( $\left.\mathrm{n}^{\mathrm{o}} 2\right)$, en La Carolina; y El Berrueco ( $\left.\mathrm{n}^{\circ} 14\right)$, en Torredelcampo. Donde sí han existido documentamos tres tipos de actividades arqueológicas: la prospección, el estudio paramental (exclusivo o junto con otros métodos), y la excavación (parcial o total). Son cuatro las prospecciones arqueológicas llevadas a cabo, en 2003 se intervino en la Peña de Martos (Fig. 1.17), (Beatriz et al., 2009). Con posterioridad se actuó en el castillo de Giribaile (Fig. 1.4) en 2004 (Castillo, 2011), con una metodología más sistemática. Esta último trabajo marcó las directrices usadas posteriormente en el castillo de Vilches (Fig. 1.3) en 2006 (Gutiérrez, 2014); y en el castillo de la encomienda del Víboras (Fig. 1.20) en 2008 (González, 2009). Respecto a los estudios paramentales fueron exclusivos en el exterior del castillo de la Tobaruela (Cid, 2018); y junto con otras técnicas en el castillo de Santa Catalina (Jaén), o en el castillo de Lopera.

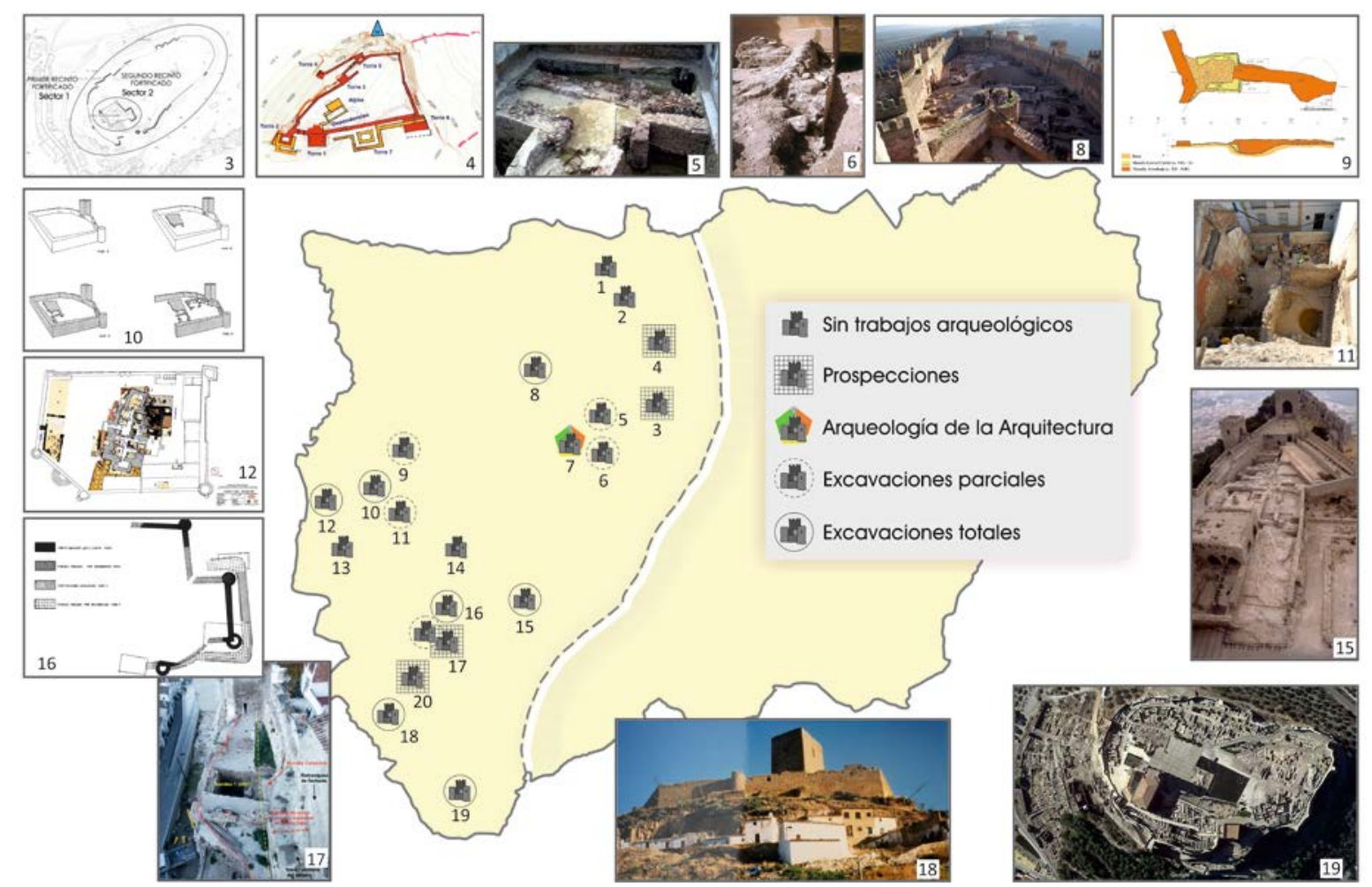

Fuente: Mapa (Carmona, 2019). Láminas: 3 Gutiérrez (2014), 4 Castillo (2011), 5 Téllez (2015), 6 VV.AA. (1997), 8 Propia, 9 Palomino (2015), 10 Nocete (1990), 11, 12 y 15 Archivo personal de J.C. Castillo, 16 Lizcano (1993), 17 Serrano (2017), 18 Salvatierra (1994), y 19 Google Maps

Fig. 1 Estado actual de las fortificaciones de la ruta respecto a actuaciones arqueológicas

Finalmente, las excavaciones puntuales o en extensión, son las intervenciones más repetidas. Entre las primeras encontramos las realizadas en diferentes lugares del recinto amurallado de Andújar (Fig. 1.9), desde 1989 (Palomino, 2015); de apoyo a la restauración como en la torre de Santa Eufemia (Fig. 1.6), en Cástulo (Linares), en 1993 (VV.AA., 1997), o en la Torre Almedina (Fig. 1.17), de la villa de Martos (2007 - 2015) (Serrano, 2017). También existen otras realizadas de urgencia en el castillo de Linares (Fig. 1.5), en 2011 (Téllez, 2015); o en un proyecto más amplio como en Arjona (Fig. 1.11) a corto (2016 - 2018) y a largo plazo para recuperar un amplio tramo de muralla como recoge la APP "Murallas de Arjona". Las fortificaciones excavadas en extensión han sido siete desde 1998 que se intervino (Nocete, 1990), en Arjonilla (Fig. 1.10); seguida, entre 1992 y 1993 (Castillo y Marín, 1999), por Santa Catalina en Jaén (Fig. 
1.15), condicionada por los posibles futuros usos (Castillo, 2014). En 2002, se realizó una intervención en Baños de la Encina (Fig. 1.8) ante el peligro que suponía la colmatación de los sedimentos en su interior, refutando el falso histórico de castillo califal y datándose en época almohade (Moya, 2014). Otro excavado en gran parte entre 1990 y 1992 (Lizcano, 1993), pero con zonas aún sin excavar, es el castillo de Torredonjimeno (Fig. 1.16). Inacabados por su entidad se encuentran los trabajos en la fortaleza de la Mota (Fig. 1.19), realizados de manera casi ininterrumpida desde 1990 (VVAA., 1992) hasta la actualidad. Conocemos por las excavaciones realizadas entre 1992 y 2007 (Anguita, 2011), las fases de los castillos de Alcaudete (Fig. 1.18): hịnn islámico, encomienda calatrava, palacio renacentista; y de Lopera (Fig. 1.12): dos fases de iglesias, el uso de iglesia fortificada, de vivienda palaciega y de bodega, documentadas en los trabajos realizados entre 2007 y 2011 (Pantoja, 2011).

\section{Conservación actual}

Hay factores que influyen en el estado de la arquitectura defensiva: físicos con la vulnerabilidad propia de los materiales constructivos; el paso del tiempo y la afección de elementos climáticos, que llevan a la generación de patologías específicas y concretas, como la carbonatación, que se refleja en la erosión del castillo de Castro Ferral (Fig. 2.1), de las murallas de Andújar (Fig. 2.9) o la fragmentación, dependiendo del material sobre el que influya. El agente más dañino es el ser humano, con la destrucción deliberada, actuaciones sin control y actos vandálicos diversos como grafitis (Fig. 2.17A izq.). Para conocer la situación actual nos adentraremos, además de en la observación directa, en los trabajos de consolidación y restauración: cómo se han realizado, y si han contado o no con equipo interdisciplinar.

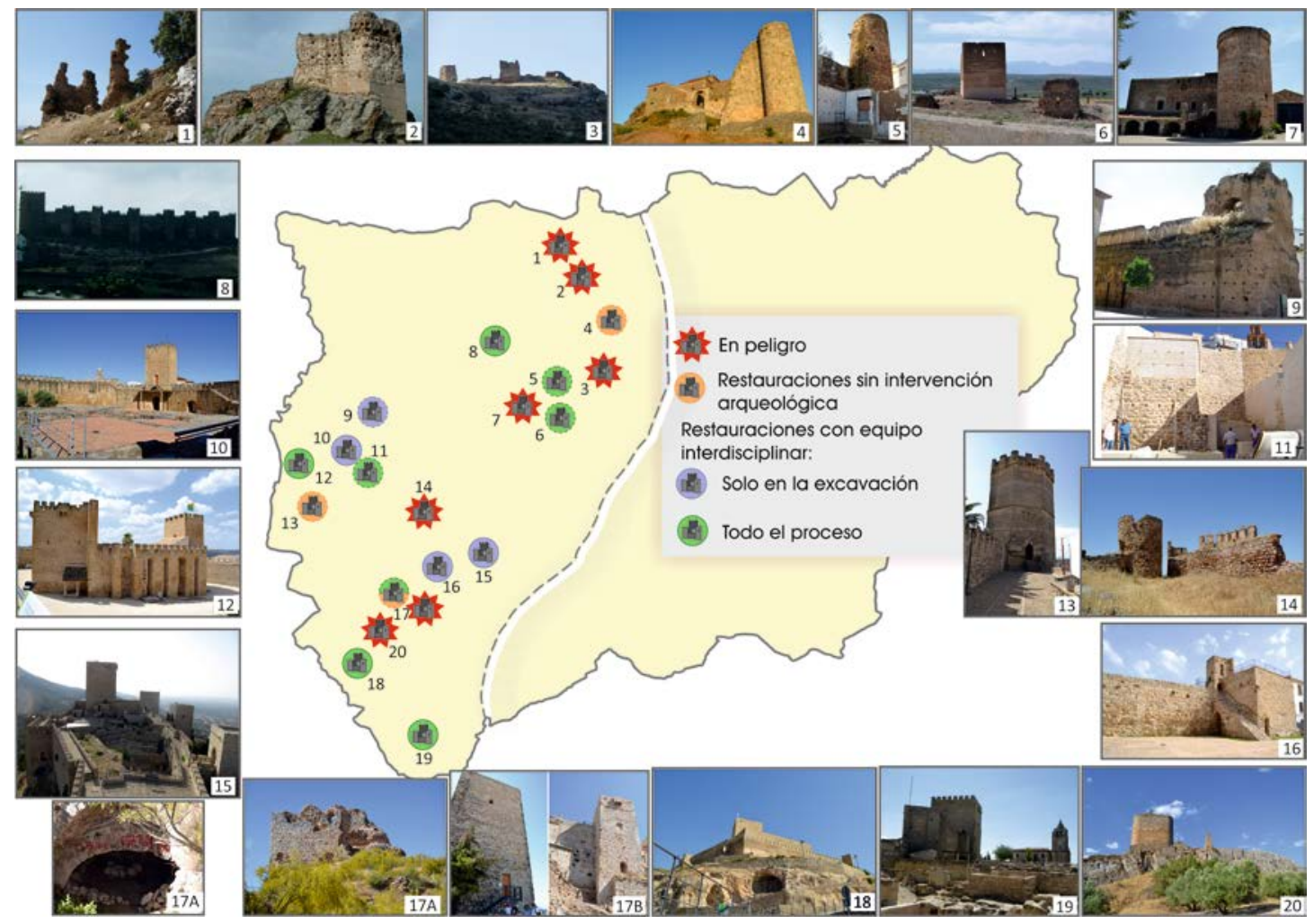

Fuente: Mapa (Carmona, 2019). Láminas: Archivo personal, excepto láminas 2 y 5 del Archivo personal de J. C. Castillo

Fig. 2 Actuaciones de restauración realizadas

\subsection{Restauraciones en fortificaciones: presencia de investigaciones y formación de equipos interdisciplinares}

Las intervenciones de restauración no siempre están basadas en investigaciones previas, como en la restauración de la Torre de Boabdil (Fig. 2.13), de Porcuna, en 1976, con la reconstrucción de los muros perimetrales, y de las almenas (Anguita, 2011); o la realizada en la Torre del Homenaje (Fig. 2.17B izq.) del castillo de la villa de Martos, en 1982 (Anguita, 2011). Más recientes son los trabajos de conservación puntuales en parte del recinto amurallado de Andújar: 
recubriendo la parte superior para evitar la erosión en las torres de la calle Silera o con la actuación de emergencia en el tramo de la calle Luis Vives para su consolidación, que se han ejecutado con criterios básicos como la compatibilidad de los materiales con la estructura, la reversibilidad y neutralidad de las actuaciones entre otras.

Cuando se han realizado intervenciones tras una excavación arqueológica, en la mayoría de las ocasiones la comunicación entre arquitectos y arqueólogos, no ha sido todo lo fluida que debiera. Esto sucedió por la inexistencia de un equipo multidisciplinar obligado a escuchar y llegar a acuerdos entre las partes con contacto en todas las fases de actuación. Por tanto, la relación en algunos casos fue escasa dificultándose el entendimiento y primando criterios propios ante las actuaciones en los castillos. Esto ocurrió por el paso del tiempo en el castillo de Arjonilla (Fig. 2.10), o en las acciones realizadas desde la Escuela Taller Municipal de Torredonjimeno para restaurar la torre y el lienzo de muralla (Fig. 2.16).

En contraposición, otras intervenciones han sido efectuadas con la presencia de un equipo multidisciplinar como en Alcaudete (Fig. 2.18), entre 1997-2006, donde restauraron las puertas, bestorres, adarves, cuerpos de guardia, el aljibe mayor, el refectorio y la Torre del Homenaje. Otro ejemplo, son las intervenciones, entre 2006 y 2009, en el castillo de Lopera (Fig. 2.12) que no solo reforzaron la estructura y la techumbre, sino que consolidaron los restos más significativos. En Baños de la Encina (Fig. 2.8), entre 2011 y 2015, se reforzaron y restauraron algunas torres, y consolidaron las estructuras halladas en la excavación. La fortaleza de la Mota (Fig. 2.19) representa un enorme trabajo continuado desde los años 70 y 80, restaurando el Alcázar, la Iglesia Mayor Abacial, la Muralla Norte y la torre de la Cárcel, obteniendo el Premio a la Conservación del Patrimonio como factor de desarrollo económico y social, en 2017. Actualmente (febrero 2020) se está finalizando el proyecto que comenzó en 2018 de restauración de la Torre Almedina del castillo de la villa de Martos (Fig. 2.17B dcha.). En septiembre de 2019, concluyeron las obras en los restos del lienzo sur y torreón del Alcázar de Arjona (Fig. 2.11), siguiendo para el recrecimiento de los muros técnicas similares a las de la época con la realización de tapial con encofrado de madera, y ejecutado en base a la vara castella y sus medidas.

\subsection{Fortificaciones sin actuaciones}

Aún no se ha realizado intervenciones que ayuden a su conservación en siete de las fortificaciones analizadas: el castillo de Castro Ferral (Fig. 2.1); el de las Navas (Fig. 2.2); el castillo de Giribaile (Fig. 2.3) incluido en la Lista roja de Patrimonio de Hispania Nostra; el castillo de la Tobaruela (Fig. 2.7) con un uso continuado como vivienda. Uno de los castillos que ha tenido enormes reivindicaciones para que se actúe es el castillo de El Berrueco en Torredelcampo (Fig. 2.14), para el que recientemente se ha aprobado la ejecución de una obra de emergencia. Con un estado semejante, pero sin intervenciones previstas se encuentran los castillos de la Peña de Martos (Fig. 2.17A), también en la Lista roja de Patrimonio; y el castillo de La Encomienda del Víboras en Martos (Fig. 2.20). Para todos ellos, recomendamos un especial seguimiento de las estructuras y actos vandálicos más prolíficos por su lejanía con los centros urbanos.

\section{Musealización y difusión}

\subsection{Señalización y Musealización}

No todas las fortificaciones tienen señalética básica de la ruta: Castro Ferral $\left(\mathrm{n}^{\circ} 1\right)$ y las Navas $\left(\mathrm{n}^{0} 2\right)$ por encontrarse en el Parque Natural de Despeñaperros y en una finca privada; Linares $\left(\mathrm{n}^{\circ} 5\right)$ por ser de difícil acceso y visibilidad reducida desde el entorno. El resto de las fortificaciones tienen la básica de la ruta (Fig. 3), compuesta por un tótem en las vías de acceso, alternando otro general de la población, o el específico de cada castillo según las necesidades del bien. Varían en tres lugares: en el castillo de Tobaruela $\left(\mathrm{n}^{\circ} 6\right)$, existente únicamente el de las vías de acceso por su titularidad privada; en Andújar donde se alterna con paneles propios - no anclados de la manera más adecuada- con los agarres de hierro de uno de estos incrustado directamente en el tapial (Fig. 3.9); o en Torredonjimeno donde la señalética propia aúna la de la ruta en paneles individualizados en cada uno de los elementos puestos en valor.

La musealización de estos espacios ha llevado a la consolidación de diferentes espacios destinados a centros de interpretación, como el de la Batalla de las Navas de Tolosa, que sirve de introducción al visitante para el conocimiento 
histórico de la zona; el Arqueológico de Giribaile $\left(\mathrm{n}^{\circ} 3\right)$ con un espacio dedicado a la fase medieval del yacimiento; o el Cultural e Histórico de Martos en el interior de la Torre del Homenaje ( ${ }^{\circ}$ 17), que abarca la evolución histórica de la ciudad. Son cuatro los castillos convertidos en centros de interpretación en su totalidad, siendo el primero en construirse el de Santa Catalina en Jaén (Fig. 3.15), que presenta deficiencias por la instalación sin los trabajos previos de consolidación y restauración, funcionando mal los elementos electrónicos. Uno de los más completos es "La vida en la frontera" (Fig. 3.19), de la Fortaleza de la Mota (Alcalá la Real), con gran cantidad de aspectos de las sociedades que lo habitaron. El de la Orden Militar de Calatrava en Alcaudete (Fig. 3.18), destaca por su accesibilidad salvo en la Torre del Homenaje. El último en inaugurarse ha sido el homónimo de Lopera (Fig. 3.12), con gran claridad expositiva, muy didáctico y que representa, como sus antecesores, la historia del castillo. La mayor crítica a éste, es su mal uso desvirtuando la historia con la escultura de un musulmán que hace de vigía en un castillo de época cristiana; siendo el mayor acierto la concienciación de la importancia de la labor de los profesionales que lo han recuperado.

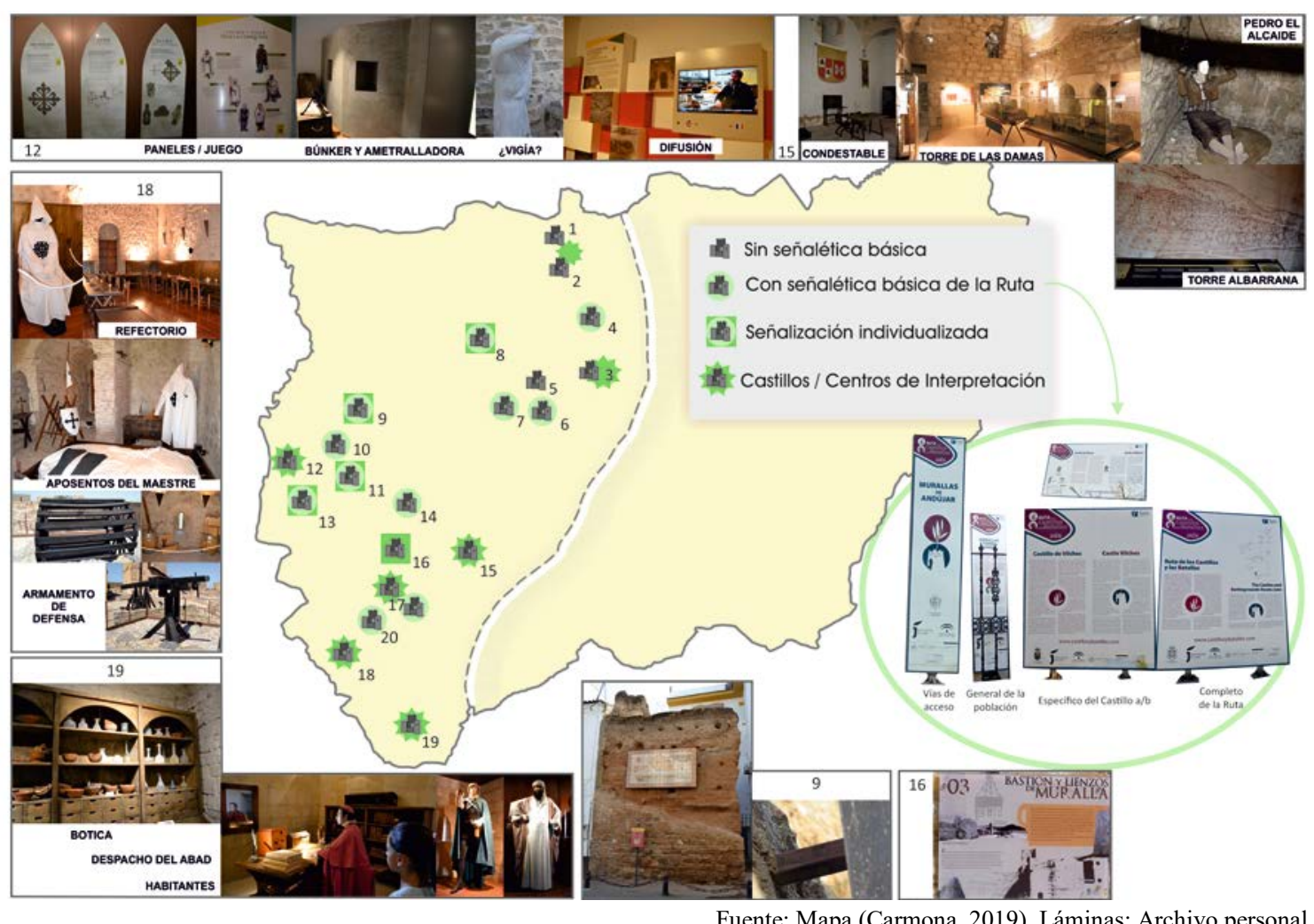

Fig. 3 Tipos de señalética o musealización en los castillos o recintos amurallados de la ruta

\subsection{Difusión}

Dentro de las actividades fomentadas dentro de la ruta diferenciaremos entre: las grandes y únicas en cada municipio, con importante aportación municipal; y las idénticas que se repiten por los municipios de la ruta. Respecto a las primeras, hallamos algunas consagradas como las Veladas Nazaríes de Arjona, las Fiestas Calatravas de Alcaudete, la Noche Andalusí de la Rosa en Baños, junto con otras que están aún en los primeros pasos como la Marcha de los Tres Reyes o la Recreación de la Batalla de las Navas de Tolosa, muestras indiscutibles del interés y apuesta por la atracción del turismo. Estas coexisten con otras enfocadas a un público local, idénticas entre sí: mercados medievales, torneos de exhibición de combate medieval, Astroturismo... claves en la difusión de la ruta en 2019. Esenciales son las actividades enfocadas a los discentes que pretenden concienciar y sensibilizar al alumnado desde edades tempranas.

Junto con las grandes actividades los municipios pueden promocionar sus bienes a través de páginas webs y redes sociales (para toda la localidad; del conjunto del patrimonio municipal o específicas del castillo). Otro elemento que se está abriendo paso es el uso de APP para dispositivos móviles, que incluyen digitalizaciones del patrimonio como en los castillos de Baños de la Encina y Torredonjimeno, o en la muralla del Alcázar de Arjona. 
Los profesionales e investigadores debemos también difundir el conocimiento, revertiendo de nuevo en la sociedad, dirigiéndonos a otros profesionales para enriquecer los conocimientos mutuos, pero sin olvidar al público general, siendo esencial su sensibilización, mediante jornadas de puertas abiertas, exposiciones, visitas guiadas, conferencias...

\section{A modo de conclusión: debilidades y fortalezas. Propuestas de actuaciones}

Hemos realizado un decálogo de actuaciones para mejorar las debilidades y fomentar las fortalezas como el seguimiento de las estructuras, especialmente aquellas que no han tenido programas de conservación/restauración, para evitar daños que causen pérdidas irreparables. También vemos imprescindible el cumplimiento de las garantías mínimas en todas las intervenciones que se lleven a cabo: todo lo que no documentemos hoy, mañana no podremos recuperarlo para su estudio y posterior puesta en valor. Proponemos una revisión y actualización de los contenidos de la web oficial, eliminando los puntos no visibles normalmente, adaptando los horarios, incorporando el centro de interpretación de Giribaile y alertando de los peligros de algunas visitas. En algunos castillos mejoraría las señales de acceso y ampliaría la información in situ con información complementaria de los estudios ya realizados sobre algunos, sobre todo en los ubicados en ámbitos rurales. Respecto a la difusión focalizaría las actividades en señas de identidad propias (Carmona, 2019).

La Ruta de los Castillos y Batallas supuso, en 2017, el 48\% de los turistas que visitaron la provincia (Martín, 2018), variando el repartimiento de los flujos turísticos entre unos y otros, por motivos como la visibilidad, presencia de trabajos arqueológicos, el estado en los tres vértices analizados, los tiempos de apertura... Variando en 2018 desde los 45124 visitantes de la Mota hasta los 728 de Arjonilla. Finalmente proponemos una ampliación de la ruta (Fig. 4) ampliando la ya existente y creando nuevos ejes hacia el Condado (eje 1), la Loma y la sierra de Segura y Cazorla (eje 2), y sierra Mágina (eje 3). No olvidamos que Jaén es la provincia con más fortificaciones por kilómetro cuadrado de Europa por lo tanto, somos conscientes que es denso el camino andado, pero aún queda mucho por recorrer (Carmona, 2019).

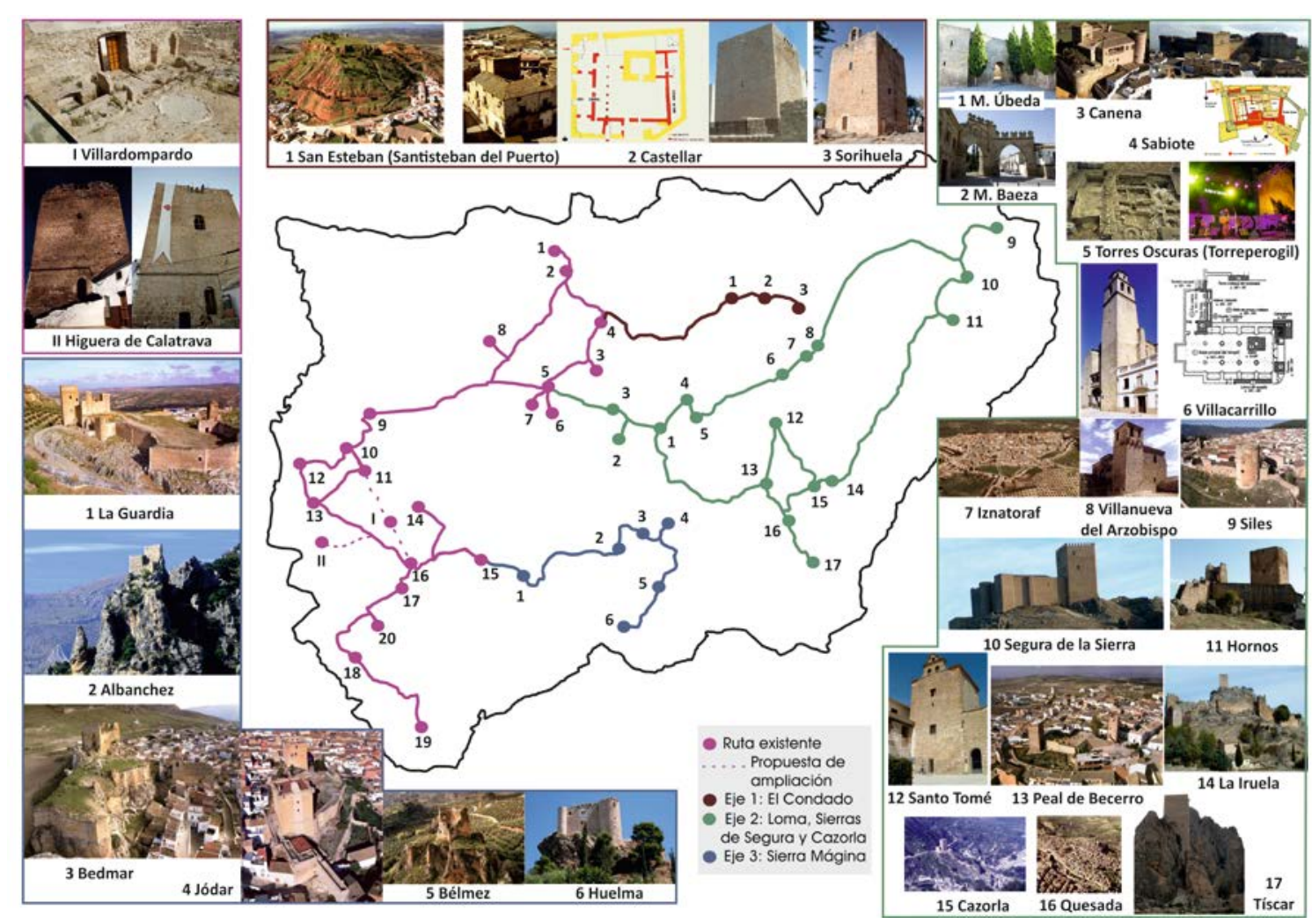

Fuente: Mapa de elaboración propia. Láminas: Archivo personal de J.C. Castillo

Fig. 4 Propuesta de ampliación y nuevos ejes de la Ruta de los Castillos y las Batallas 
El patrimonio arquitectónico defensivo giennense: investigación, difusión y puesta en valor. Actuaciones realizadas en las fortalezas que integran la Ruta de los Castillos y las Batallas

\section{Referencias}

Anguita, M. R. (2011). Conservación y restauración de la arquitectura calatrava en la provincia de Jaén. En M. V. Gómez (Ed.), La arquitectura de las órdenes militares en Andalucía: conservación y restauración (pp. 323-371). Huelva: Universidad de Huelva.

Beatriz, M., Serrano, J. L., y Cano, J. (2009). Prospección arqueológica superficial con recogida de material y limpieza en la Peña de Martos (Jaén). Anuario Arqueológico 2004(1), 2006-2011.

Carmona, A. M. (2019). El patrimonio arquitectónico defensivo giennense: investigación, difusión y puesta en valor. Actuaciones realizadas en las fortalezas que integran la Ruta de los Castillos y las Batallas (Trabajo Fin de Máster). Universidad de Jaén, Jaén.

Castillo, J. C. (2014). De la investigación a la difusión: el caso del Castillo de Santa Catalina en Jaén. En C. Sánchez, L. Pérez y S. Rodrigo (Eds.), Los castillos: reflexiones ante el reto de su conservación (pp. 251-269). Sevilla, España: Consejería de Cultura de la Junta de Andalucía.

Castillo, J. C. (2011). El asentamiento islámico de Giribaile. En L. Gutiérrez (Ed.), Guía arqueológica de Giribaile (pp. 330-396). Torredonjimeno: Águila.

Castillo, J. C., Castillo, J. L., Marín, M. M., y Pérez, M. C. (1999). La Arqueología de apoyo a la restauración: teoría o realidad. El caso de las fortificaciones giennenses. En D. Armada (Coord.), Arqueología del Monumento. Actas de los III Encuentros sobre Arqueología y Patrimonio (pp. 205-230). Salobreña: Ayuntamiento de Salobreña.

Castillo, J. C., y Marín, M. M. (1999). Informe técnico sobre los trabajos de excavación y consolidación arquitectónica en el castillo de Santa Catalina (Jaén). Anuario Arqueológico de Andalucía, 1994, Tomo III Urgencias, 259-270.

Cid, C. (2018). Fotogrametría y estudio histórico-constructivo del Castillo de la Tobaruela (Trabajo de Fin de Máster). Universidad de Jaén, Jaén, España.

Eslava, J. (1999). Los Castillos de Jaén. Granada, España: Osuna.

González, J. (2009). El Conjunto Fortificado de Víboras: una aproximación a su organización espacial (Trabajo final de estudios avanzados. Tesina). Universidad de Jaén. Jaén.

Gutiérrez, M. V. (2014). La evolución de un conjunto fortificado: el protagonismo de Vilches entre los siglos XII y XIII. En P. Cressier y V. Salvatierra (Eds.), Las Navas de Tolosa 1212-2012: miradas cruzadas (pp. 551-566). Jaén: Universidad de Jaén.

Lizcano, R., Aguayo, M., Araque, D., Hernández, M., Izquierdo, M., y Ruiz, J. (1993). 2a campaña de excavaciones arqueológicas en el Castillo de Torredonjimeno (Jaén). Anuario Arqueológico de Andalucía 1991, tomo III, 305-311.

Madueño, J. J. (2017). Jaén, la provincia española con más fortalezas. Retrieved August 3, 2019, from https://www.abc.es/viajar/destinos/espana/abci-jaen-provincia-espanola-mas-fortalezas-201701192051 noticia.html

Martín, A., Fernández, J. P., y Herrador, I. (2018). Análisis del sector turístico de la provincia de Jaén, de sus macrozonas, comarcas y de la Ruta de los Catillos y las Batallas (Inédito). Instituto de Estudios Giennenses y Universidad de Jaén.

Moya, S. (2014). Actuación arqueológica puntual en el Castillo de Burgalimar de Baños de la Encina (Jaén), 2007-2009. En P. Cressier y V. Salvatierra (Eds.), Las Navas de Tolosa 1212-2012: miradas cruzadas (pp. 593-606). Jaén: Universidad de Jaén.

Nocete, F. (1990). Memoria de Actuación Arqueológica Urbana en el castillo de Arjonilla. Anuario Arqueológico de Andalucía 1988, tomo III, 182-190.

Palomino, J. A. y Castillo, J. C. (2015). Transformaciones urbanisticas de Andújar a través de la muralla medieval. Jaén, España: Diputación Provincial de Jaén, Instituto de Estudios Giennenses.

Pantoja, J. L. (2011) Fin de la excavación en el Castillo de Lopera. Retrieved August 11, 2019, from https://www.diariojaen.es/historico/fin-de-la-excavacion-en-el-castillo-de-lopera-LODJ39278

Salvatierra, V., y Castillo, J. C. (1994). Carta de Riesgo de Alcaudete (Proyecto de investigación inédito). Instituto de Estudios Giennenses y Universidad de Jaén.

Serrano, J. L, y Cano, J. (2017). Memoria histórico-arqueológica. Proyecto de Rehabilitación de la Torre Almedina, Lienzos de Muralla y entorno inmediato en Martos (Fase III). Martos: Ayuntamiento de Martos.

Téllez, I. (2015). El Ayuntamiento rechaza la propuesta para adecuar los vestigios del Castillo de Linares. Retrieved August 5, 2019 , from: https://www.ideal.es/jaen/linares/201508/26/ayuntamiento-rechaza-propuesta-para-20150825235642.html

VV.AA. (1997). Anuario Arqueológico de Andalucía 1993. Tomo I, Sumario, 37. 